\title{
Low temperature two STM tip tunneling measurements of a floating chemical potential $\mathrm{Pb}(111)$ surface
}

\author{
We-Hyo Soe ${ }^{1,2, *}$, Corentin Durand ${ }^{1}$, and Christian Joachim ${ }^{1,2}$ \\ ${ }^{1}$ CEMES, Université de Toulouse, CNRS, 29 Rue J. Marvig, BP 94347, 31055 Toulouse Cedex, France \\ 2 International Center for Materials Nanoarchitectonics (WPI-MANA), National Institute for Materials Science (NIMS), Namiki, \\ Tsukuba, Ibaraki 305-0044, Japan
}

Received: 10 April 2019 / Received in final form: 4 September 2019 / Accepted: 1 October 2019

\begin{abstract}
On a $\mathrm{Pb}(111)$ superconducting surface, low temperature $\mathrm{d} I / \mathrm{d} V$ tunnelling spectra are recorded between two scanning tunnelling microscopes ( $\mathrm{STM}$ ) metallic tips with the $\mathrm{Pb}(111)$ sample metallic support non-grounded. The tunnelling current intensity I passing between the 2 tips through the sample is controlled by changing one or both STM vacuum tunnelling junction resistances. The chemical potential of this floating $\mathrm{Pb}(111)$ surface depends on the normalized ratio between those two quantum resistances. When ungrounded, the $\mathrm{Pb}(111)$ sample chemical potential balances between those of the 2 STM tips while tuning their respective tip end atomic apex to $\mathrm{Pb}(111)$ surface distances with a picometer precision without any physical contact between the STM tips and the surface.
\end{abstract}

\section{Introduction}

In a multiple contact electrodes set-up fabricated on the surface of a conductive material to characterize, for example, its intrinsic electronic transport properties, the different chemical potentials of the source, drain and/or floating electrodes are governing the current intensities passing through the sample [1]. Current intensities also depend on the contact resistances (Ballistic, Ohmic, Schottky) between the electrodes and the sample, especially when the sample size is smaller than the electronic mean free path of the material $[1,2]$. The contact electrodes are usually fabricated using micro and nanofabrication techniques [3]. Their positioning onto the sample surface is fixed (one multiple contacts configuration per fabrication run) and located at best with a few nanometres precision on this surface $[4,5]$. The precision is generally less due to the lateral diffusion of the very last metal atoms during the electrodes metallization step [6] either using a resist, a shadow mask $[7,8]$ or a dynamic stencil [9].

Recently, multi-probes scanning tunnelling microscopes (STM) have been developed to enable a movable and more precise way of positioning the contacting electrodes. Here and on the sample surface, the lateral and vertical positioning of the STM tip electrodes is controlled within a few nanometers. More important, the contact resistances can be adjusted using the STM tip-tosurface vertical distance [10-14]. Without touching physically the surface, it opens the way to control the

\footnotetext{
* e-mail: we-hyo.soe@cemes.fr
}

elementary electron transfer processes from the tip end apex to the sample surface (or the reverse) at the origin of the measured tunnelling current intensity.

Such a tunnelling contact is obtained when the STM tip end atom is maintained further away from the surface uppermost atom than the van der Walls distances. There is no physical deformation of the surface nor chemical bond to the surface. In this case and when the sample is not grounded, its surface is set in an electronic floating configuration with still electrons able to be transferred from one STM tip to another through the surface. In this configuration explored below, there is a non-classical potential drop due to decoherence effects at each tip apex to surface vacuum tunnelling junction. This strictly noninvasive measurement configuration is to be found when the electronic orbitals overlap between the sample surface and the STM end atom tip apex are maintained as small as possible while conserving long range electron transfer events between the tips through the surface. This configuration is also to be found when measuring the intrinsic conductance of a surface atomic wire [15] or a molecular wire [16] without perturbing their molecular orbitals [17].

To reach this strictly non-invasive tunnelling measurement configuration, a new very stable low temperature (LT) ultra-high vacuum (UHV) four STM scanners instrument (LT-UHV 4-STM) [18] is used to measure the chemical potential of an atomically clean UHV prepared $\mathrm{Pb}(111)$ sample in a $2+1$ contacts configuration. The 2 contacts are non-invasive tunnelling contacts as defined above and the third one is a macroscopic very low ohmic contact resistance at the back of the $\mathrm{Pb}(111)$ sample. The first electrode (the source, Tip A) is one atom sharp STM tip apex of the LT-UHV 4-STM. It is not physically 
nor electronically touching the surface. Using an external voltage source, it is maintained at a well-controlled electronic chemical potential $\mu_{A}=E_{F}+e V_{A}$ where $E_{F}$ is the tip metal material electronic Fermi level. The second electrode (the drain, Tip B) is a second STM tip apex (Tip B) of the same instrument. It is also not touching the surface and set at a well-controlled chemical potential $\mu_{B}=E_{F}+e V_{B}$. The third electrode is static: the underlying stainless steel $\mathrm{Pb}(111)$ sample holder on the LT-UHV 4-STM. This holder can be automatically and in situ connected to (or disconnected from) a well-defined macroscopic ground as discussed formally in [19]. When not grounded, the "lead-stainless steel bulk" chemical potential $\mu_{S}$ is floating between $\mu_{A}$ and $\mu_{B}$. As measured in this paper, $\mu_{S}$ depends critically on the atomic scale Tip A and Tip B vacuum tunnelling contacts resistance to the $\mathrm{Pb}$ (111) surface. We demonstrate how $\mu_{S}$ can be tuned in real time by setting the two STM tips distance from the $\mathrm{Pb}$ (111) surface with a few picometers precision on our LTUHV 4-STM required to master those non-invasive tunnel contacts.

\section{Preparation of the floating two tips surface measurements}

The clean $\mathrm{Pb}(111)$ surface of the lead sample mounted on its stainless steel holder was prepared following a multiple cycles of $\mathrm{Ar}^{+}$surface sputtering and $160^{\circ} \mathrm{C}$ annealing in the UHV preparation chamber of our Scienta-Omicron low temperature LT-UHV 4-STM [18]. The $\mathrm{Pb}(111)$ sample was transferred in UHV onto the sample stage and cooled down to liquid helium ( $\mathrm{LHe}$ ) temperature, below the superconducting transition temperature of lead $(T c=7.19 \mathrm{~K})$. The superconducting $\mathrm{Pb}(111)$ surface was preferred to a standard non-superconducting metal surface to follow the $z_{A}$ variations using the well characterized $\mathrm{Pb}(111)$ superconducting gap observable in $\mathrm{d} I / \mathrm{d} V$ curves.

A typical LT-UHV STM image recorded using one scanner of our LT-UHV 4-STM is presented in Figure 1a. The dark hexagonal features originate from the quantum well electronic states created between the topmost $\mathrm{Pb}(111)$ surface and the sub-surface Ar bubbles formed during the $\mathrm{Pb}(111)$ surface preparation [20]. The preparation of the atomic scale STM tip apex with a single end atom was performed by a soft indentation in the $\mathrm{Pb}(111)$ surface of each STM tip before the measurements (Fig. 1b). At LHe temperature, it certainly results in a wetting of the tungsten tip apex with a few $\mathrm{Pb}$ atoms. All our tunnelling junctions formed by this gentle indentation are still superconducting-vacuum-normal metal junctions, the lead layer thickness captured during the tip indentation remaining at the atomic scale. The $\mathrm{d} I / \mathrm{d} V$ spectrum of such a junction is presented in Figure $1 c$ with the $\mathrm{Pb}(111)$ sample macroscopically back grounded through the sample holder. It demonstrates a clear superconducting gap which is reproducible with all tips on the LT-UHV 4-STM and on any atomically flat terrace of the $\mathrm{Pb}(111)$ surface. According to this measurement, an effective $6 \mathrm{~K}$ tunnel junction temperature can be estimated [21].
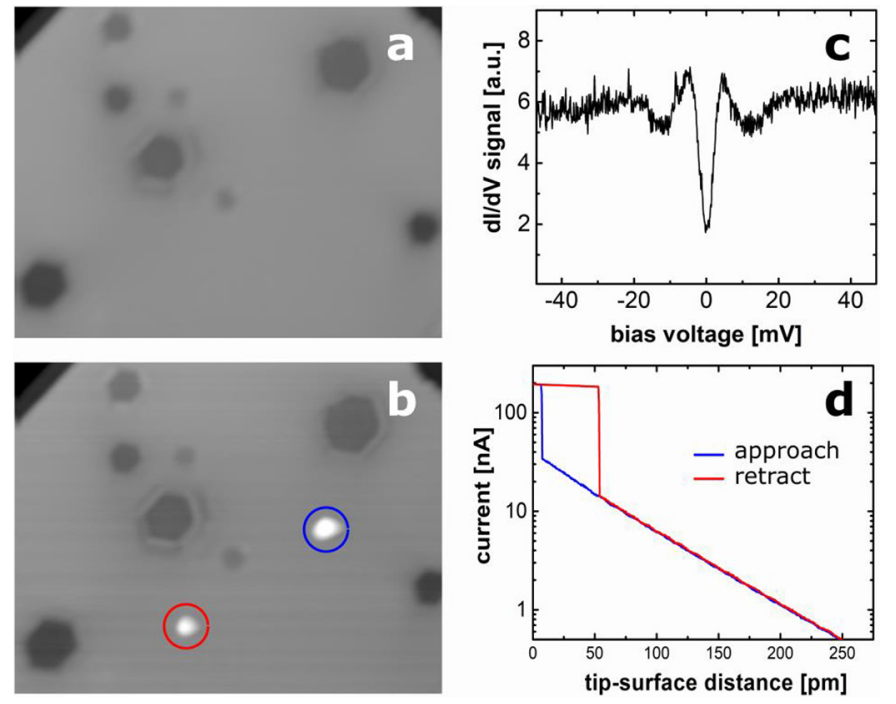

Fig. 1. Typical constant current $\mathrm{Pb}(111)$ surface images (a) before and (b) after constructing one after the other two quantum point contacts where atomic droplets have been produced while retracting the tip apex (see Fig. 1d for Tip B) with a $\mathrm{Pb}$ single atom (red circle) and a $\mathrm{Pb}$ dimer (blue circle). The hexagonal features in (a) and (b) are Ar-bubbles created during sample cleaning process in the vicinity of surface [20]. (c) Tip A $\mathrm{d} I / \mathrm{d} V$ spectrum showing the superconducting gap characteristics of a superconductor-vacuum-metal junction. Notice that the bias voltage was ramped up by of steps $0.1 \mathrm{mV}$ per $0.1 \mathrm{~s}$ for all measurements presented in this paper to avoid any low pass filter effect. (d) Two single shot current distance characteristics $I(z)$ recorded on Tip B to determine the $z_{B}$ tip apex to surface relative distance to reach the $12.9 \mathrm{k} \Omega$ quantum contact resistance. At this quantum contact, Tip B gives $I_{B}=183 \mathrm{nA}$ for $V_{B}=6 \mathrm{mV}$ i.e. an $R_{B}=32.8 \mathrm{~K} \Omega$ junction resistance $(12.9 \mathrm{k} \Omega+\mathrm{a}$ very low noise $20 \mathrm{k} \Omega$ metallic resistance in series). (a) and (b) images STM: $I=10 \mathrm{pA}, V=30 \mathrm{mV}$, images size: $40 \mathrm{~nm} \times 30 \mathrm{~nm}$. Equivalent images for Tip A and Tip B. The single one shot $I_{B}=I_{B}\left(z_{B}\right)$ were recorded in $10 \mathrm{~s}$ with the corresponding STM B feedback loop frozen. Multiple single shot $I(z)$ characteristics were recorded for Tip A and Tip B. They present all the same slope (work-function). Only the jump to contact is changing from curve to curve with a $\mathrm{z}$ shift below $50 \mathrm{pm}$.

Since the 4 STM tips of our multi-probes LT-UHV can be controlled and scanned independently on the same $\mathrm{Pb}$ (111) surface, Tip B was first set in a ballistic contact transport regime and Tip A in a tunnelling contact regime with the $\mathrm{Pb}(111)$ sample surface. To achieve this configuration, the distance $z_{B}$ between the end atom of the Tip B apex and the $\mathrm{Pb}(111)$ surface was first fine-tuned to reach one quantum of resistance $\left(R_{B}=12.9 \mathrm{k} \Omega\right)$. This was performed using the current-distance characteristics $I_{B}\left(z_{B}\right)$ recorded by anticipation with the sample holder still grounded as presented in Figure 1d, leading to a $3.45 \mathrm{eV}$ apparent tunnel barrier height [22]. This $I_{B}\left(z_{B}\right)$ curve illustrates the stability of our instrument in mastering the tip apex end atom to surface distance $>0.06 \mathrm{~nm}$ with a precision better than $2 \mathrm{pm}$ (see Ref. [18]) for mastering a strictly non-invasive vacuum tunnelling contact per tip. Then, this holder was disconnected from the ground with a Tip A tunnelling junction resistance $R_{A} \gg 12.9 \mathrm{k} \Omega$. The 

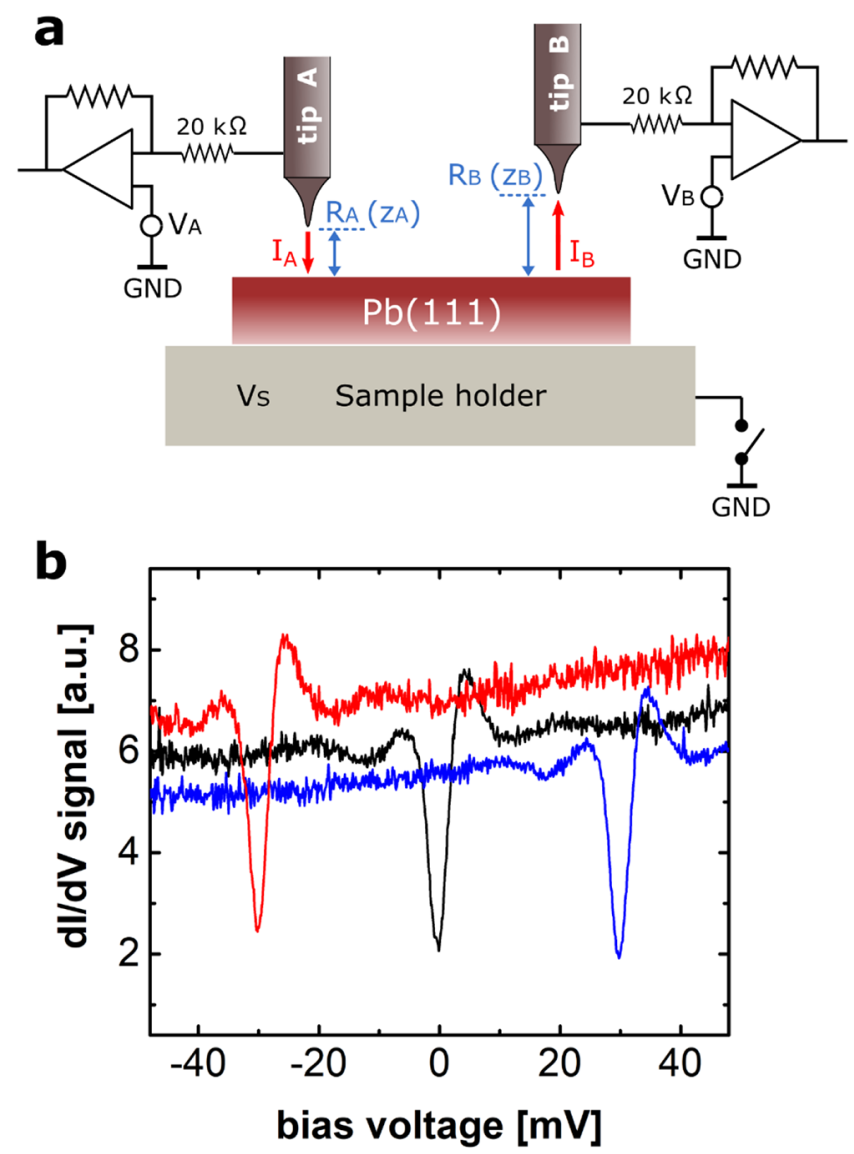

Fig. 2. (a) The two STM tip measurement electrical circuit diagram used on the $\mathrm{Pb}(111)$ surface with its 2 virtual ground $I-V$ converters and the back ground controllable switch connected to the sample holder. The $V_{S}$ sample holder is defined using $\mu_{S}=E_{F}$ $+e V_{S}$. (b) Three "one shot" $\mathrm{d} I / \mathrm{d} V$ spectra recorded on Tip A while the far away Tip B is in atomic point contact with the $\mathrm{Pb}(111)$ surface to control the sample chemical potential from its surface. Tip B potential is $V_{B}=0.0 \mathrm{mV}$ (black), $V_{B}=+30 \mathrm{mV}$ (blue) and $V_{B}=-30 \mathrm{mV}$ (red). Tip A was stabilized in a tunnelling regime with its STM Feed-back loop set up $I_{A}=200 \mathrm{pA}$, for $V_{A}=30 \mathrm{mV}$.

fine-tuned contact resistance of Tip B was preserved by freezing $z_{B}$, which is possible thanks to the stability of our LT-UHV 4-STM (lateral stability: $<0.9 \mathrm{~nm}$ per hour [18]). Its chemical potential $\mu_{B}$ was controlled by shifting the virtual ground of its STM $I-V$ converter from zero to $V_{B}=+/-30 \mathrm{mV}$ (Fig. 2b). Since $R_{B}=12.9 \mathrm{k} \Omega$, this is also fixing the $\mathrm{Pb}(111)$ surface chemical potential to $\mu_{B}$ (see below). Tunnelling $\mathrm{d} I / \mathrm{d} V$ spectra were recorded with Tip A still in a tunnelling contact junction mode on the $\mathrm{Pb}$ (111) surface. As presented in Figure $2 \mathrm{~b}$, indeed a $\mu_{B}$ change by $+/-30 \mathrm{mV}$ shifts the $\mathrm{d} I / \mathrm{d} V$ spectra accordingly with no deformation. The Tip A to Tip B apex distance can be as large as $6 \mathrm{~mm}$ on our instrument with no voltage drop along the superconducting $\mathrm{Pb}(111)$ surface while scanning Tip A with an immobile Tip B. Such $\mathrm{d} I / \mathrm{d} V$ recording certifies that the virtual ground on the Tip B STM electronics is working perfectly through the input transistors differential pair of its $I-V$ converter.

\section{Two tips measurements in a sample floating mode}

Starting from Tip A in tunnelling and Tip B in ballistic contact regimes, the distance $z_{B}$ between Tip B apex and the $\mathrm{Pb}(111)$ surface was systematically varied to change the Tip B junction resistance from this ballistic to different tunnelling junction resistances. With $V_{A}$ and $V_{B}$ set to $30 \mathrm{mV}$ and the sample holder grounded, the STM feedback loop current was first set at $I_{A}=200 \mathrm{pA}$ for Tip A and $I_{B}=2 \mathrm{nA}$ for Tip B. Once $z_{A}$ and $z_{B}$ are stabilized, the feedback loop of both STM's are opened and the sample holder disconnected from the ground. A spectroscopic measurement is then performed by sweeping $V_{A}$, while $V_{B}$ is set to zero $\left(\mu_{B}=E_{F}\right)$ through its STM $I-V$ convertor. At the end of a measure sequence, both tips and the sample are back to their initial set up and sample holder grounded with the feedback loops on. Then, the corresponding $R_{A}$ and $R_{B}$ tunnelling junction resistances are set to the next values by changing again the feedback loop current set points. As presented in Figure 3, a set of measurements correspond to the Tip B current setting reduced measurement after measurement sequences from $2 \mathrm{nA}$ to $200 \mathrm{pA}$ in 10 set points $(2 \mathrm{nA}, 1 \mathrm{nA}$, and down to $200 \mathrm{pA}$ at intervals of $100 \mathrm{pA}$ ). When reaching $I_{B}=200 \mathrm{pA}$, Tip A current was then increased from its initial $I_{A}=200 \mathrm{pA}$ up to $2 \mathrm{nA}$ following an equivalent measurement sequence now on Tip A in 10 set points.

When the sample holder ground is disconnected, Tip $\mathrm{B}$ acts as a virtual ground. The tunnelling current flows from Tip A to Tip B through the $\mathrm{Pb}(111)$ sample with $e$ $\left(V_{A}-V_{B}\right)$ the Tip A to Tip B chemical potential difference. At each $\left(I_{A}, I_{B}\right)$ combination of the feedback loops set points, a $\mathrm{d} I_{A} / d V_{A-B}$ spectrum was recorded on Tip A and then a $\mathrm{d} I_{B} / d V_{A-B}$ on Tip B using a standard lock-in technique on each junction. All $\mathrm{d} I / \mathrm{d} V$ spectra shown in Figure 3 were recorded in constant height mode and with no averaging. For reference, Figure 3(a)i and (b)i presents respectively the $\mathrm{d} I_{A} / \mathrm{d} V_{A-G S}$ and $\mathrm{d} I_{B} / \mathrm{d} V_{B-G S}$ characteristics recorded independently, both in a tunnelling contact, just before disconnecting the sample holder ground. The details of the spectrum features are different from Tip A to Tip B because of different tip apex atomic scale configurations.

For the data set selectively presented Figure 3ii to Figure 3iv and with both junction bias set up at $30 \mathrm{mV}$, a pair of set point currents $\left(I_{A}, I_{B}\right)$ corresponds here to a relative $\frac{R_{A}}{R_{A}+R_{B}}$ resistance ratio of respectively $0.91,0.83$, $0.75,0.60,0.50,0.29,0.17$, and 0.09 . The $\mathrm{d} I / \mathrm{d} V$ spectra were obtained between Tip A and Tip B while the $\mu_{S}$ sample holder is floating normally between $\mu_{A}$ and $\mu_{B}$ (see below). The spectrum recorded at the junction with the highest resistance, i.e., fixed at a larger tip apex to surface distance, shows an apparent superconducting gap close to the reference shown by Figure 3i (for example, the topmost spectrum for Tip A 3(a)ii and the bottom most spectrum for Tip B in 3(b)ii). On the contrary, spectra recorded from lower resistance junctions (smaller tip-sample distance) exhibit a progressively deformed $\mathrm{d} I / \mathrm{d} V$ spectrum with a wider apparent superconducting gap. 
a

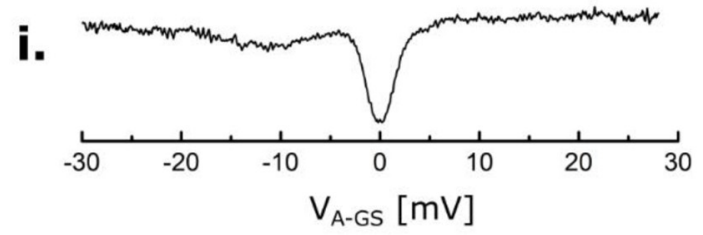

ii.

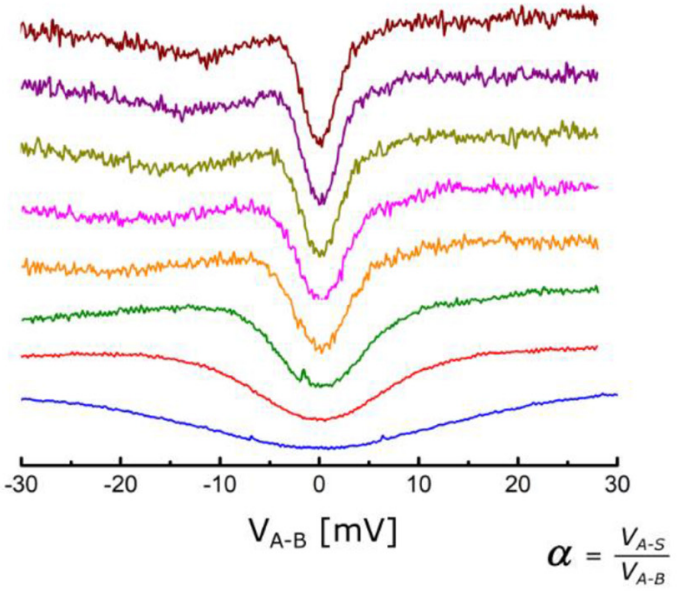

iii.
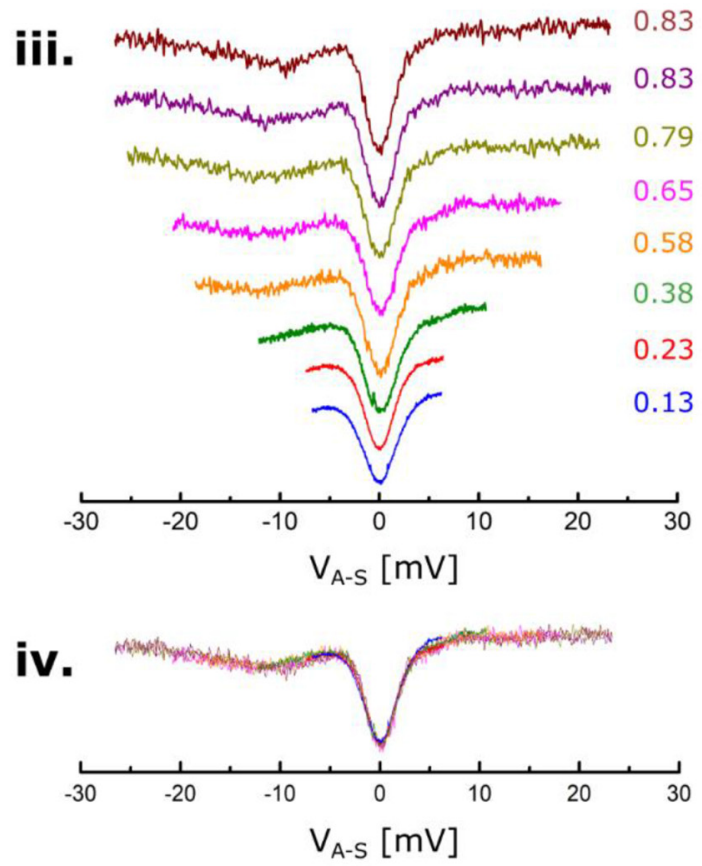

b

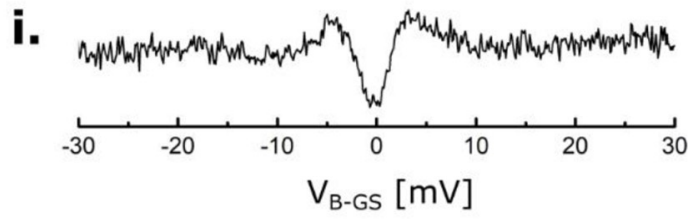

ii.

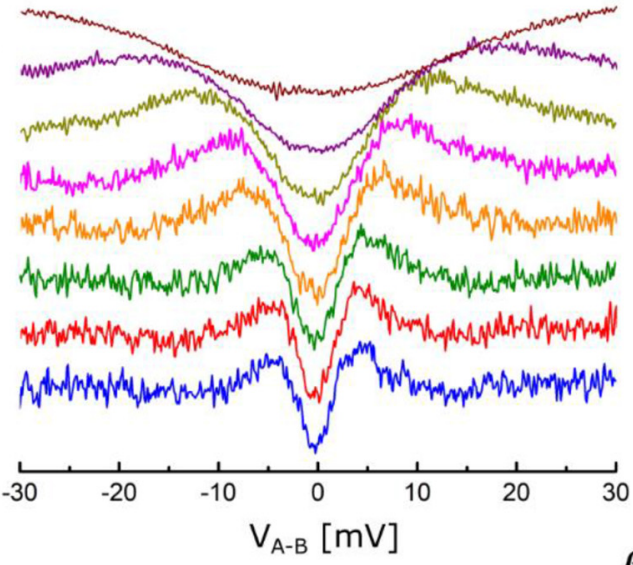

iii.
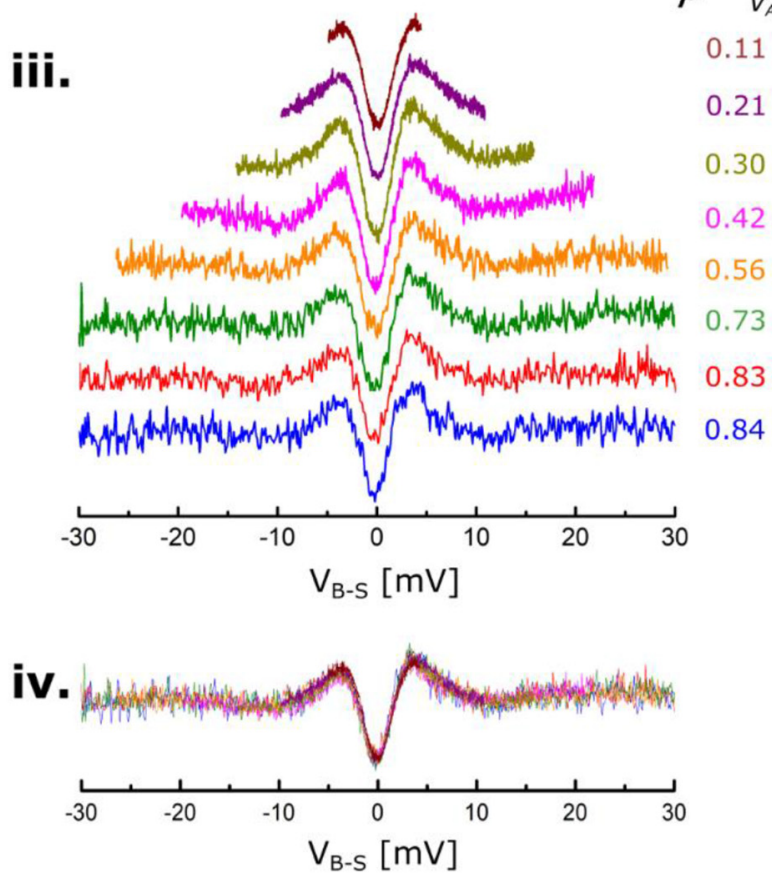

Fig. 3. A double selective series (a)ii and (b)ii of $\mathrm{d} I / \mathrm{d} V$ spectra recorded on a $\mathrm{Pb}(111)$ sample non grounded when both STM Tip A and Tip B are in tunnelling conditions. For reference, the (a)i and (b)i spectra have been recorded on Tip A and Tip B with the sample grounded. The $\alpha$ and $\beta$ parameters are obtained by fitting the (a)ii and (b)ii spectra to the (a)i and (b)i $\mathrm{d} I / \mathrm{d} V$ spectra recorded independently on the Tip A and Tip B dedicated lock-in when the sample was grounded. This fitting leads to the series (a)iii and (b)iii also perfectly superposed on (a)iv and (b)iv. $V_{A-S}=\left(V_{A}-V_{S}\right)$ the voltage difference between Tip A and the sample holder floating (see Fig. 2a), $V_{B-S}=\left(V_{S}-V_{B}\right)$ between Tip B and the sample holder floating and $V_{\mathrm{A}-\mathrm{B}}=\left(V_{A}-V_{B}\right)$ between Tip A and Tip B. $V_{\mathrm{A}-\mathrm{GS}}$ and $V_{\mathrm{B}-\mathrm{GS}}$ are the Tip A and Tip B bias voltage when the sample holder is grounded (GS). 


\section{Discussion}

To understand this apparent distortion of the measured superconducting gap when the sample holder is not grounded, the conductance $G=e I /\left(\mu_{A}-\mu_{B}\right)$ of the entire system constituted by the 2 tunnel junctions $\mathrm{A}, \mathrm{B}$ and the $\mathrm{Pb}(111)$ sample, can be written [23]:

$$
G=\left(e^{2} / \pi \mathrm{h}\right)\left(T_{A B}+\frac{T_{A S} T_{B S}}{T_{A S}+T_{B S}}\right) .
$$

$T_{A B}$ is the phase coherent transport contribution giving the number of electrons per second created by the source electrode Tip A, transferred through the tunnel barrier A, travelling in the sample between two $\mathrm{A}$ and $\mathrm{B}$ junctions without energy loss, and detected directly by the drain electrode Tip B via the tunnel junction B [19]. The second term in is a phase decoherence and energy relaxation term [23] including also for a superconducting sample the copper pair breakdown process. Here, $T_{A S}$ (respectively $T_{B S}$ ) is the transmission coefficient of the tunnelling electrons between the source electrode Tip A (resp. Tip B) and the sample through the vacuum tunnel junction A (resp. B). For this path, the transferred electrons into the superconductor sample are creating Cooper pairs at $\mu_{S}$ (i.e. losing the initial energy and phase). Those pairs are reaching the leadstainless interface, are destructed (loosing also their phase and energy) and are absorbed by the stainless steel sample holder which is in a normal metallic state. When the sample holder is not grounded, it still acts like an electron reservoir $[1,2]$. Following [23], an effective chemical potential $\mu_{S}$ can be defined for this floating reservoir leading from (1) to:

$$
\mu_{S}=\left(T_{A S} \cdot \mu_{A}+T_{B S} \cdot \mu_{B}\right) /\left(T_{A S}+T_{B S}\right) .
$$

At the drain electrode (Tip B) side, the detected electrons are created by the decomposition of Cooper pairs which are supplied by electrons coming both from A directly and indirectly from this holder reservoir. Notice that in Figure 3 presented measurement series, $T_{A B}$ was at least experimentally one order of magnitude lower than $T_{A S}$ and $T_{B S}$. This was confirmed by measuring the $I_{A B}-V_{A B}$ characteristics in the floating mode leading to $\mathrm{G}$ in (1) and this for different feedback set-up of the Tip A and Tip B junctions (see Fig. 4).

After having used those quantum electron transfer processes through the $\mathrm{Pb}(111)$ surface and the bulk to get $\mu_{S}$, it can be of interest to define an effective $V_{S}$ using the linear relation $\mu_{S}=E_{F}+e V_{S}$ indicative of the sample chemical potential shift in reference to the equilibrium $E_{F}$ (without electron transfer through the sample). Starting first from this effective $V_{S}$ definition can also be considered as a reciprocal of the approach presented in [23]. Then, $V_{A}-V_{B}$ can be written according to (2):

$$
\begin{aligned}
V_{A}-V_{B} & =\left(V_{A}-V_{S}\right)+\left(V_{S}-V_{B}\right) \\
& =\alpha\left(V_{A}-V_{B}\right)+\beta\left(V_{A}-V_{B}\right)
\end{aligned}
$$

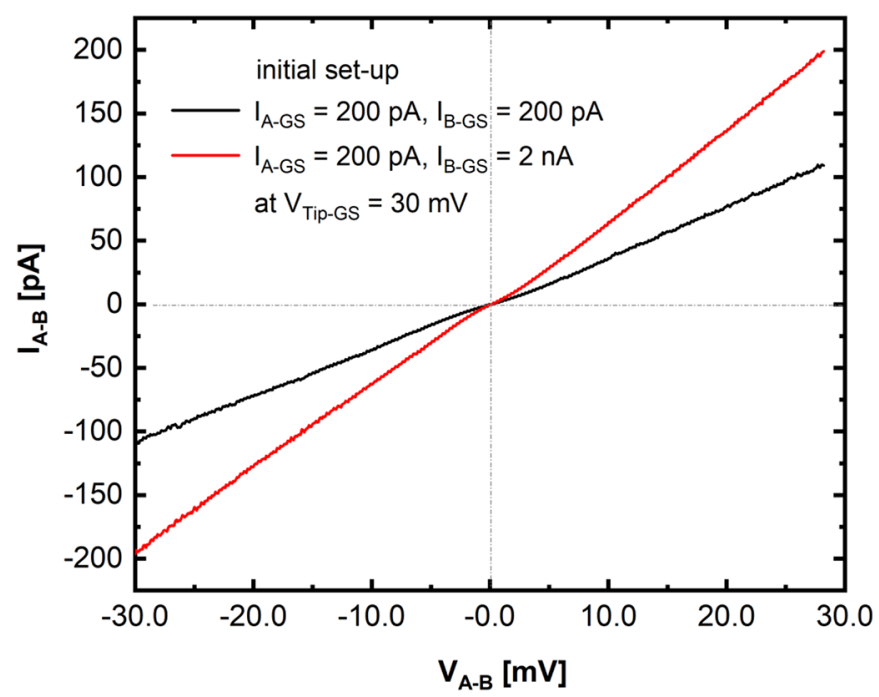

Fig. 4. Two examples of a direct floating $I_{A B}-V_{A B}$ characteristics recorded with the 2 initial set-up indicated when the sample was grounded. After setting up Tip A and Tip B distances to the $\mathrm{Pb}(111)$ surface, the sample was ungrounded. For this set up and for example in the symmetric set-up case $I_{A-G S}=200 \mathrm{pA}, I_{B-G S}=200 \mathrm{pA}$ at $30 \mathrm{mV}$, it comes for the direct to the ground $T_{A S}=0.8613 \times 10^{-4}$ (for sample grounded) and $T_{B S}=0.8613 \times 10^{-4}$ (for sample grounded). The total transmission coefficient (leading to $G$ in Eq. (1) via the Landauer formula) is $T=0.4737 \times 10^{-4}$ leading to $T_{A B}<0.043 \times 10^{-4}$ (sample not grounded).

where $\alpha=\frac{R_{A}}{R_{A}+R_{B}}, \quad \beta=\frac{R_{B}}{R_{A}+R_{B}}$ and ideally $\alpha+\beta=1$. In Figure $5 \mathrm{a}$, the experimental values of $\alpha$ and $\beta$ were determined by progressively contracting (dilating) the voltage axis of the $\mathrm{d} I / \mathrm{d} V$ curves presented in Figure 3 until they matched the reference $\mathrm{Pb}(111)$ sample holder grounded $\mathrm{d} I / \mathrm{d} V$ Figure $3(\mathrm{a}) \mathrm{i}$ or (b)i spectra. The converse behavior of the spectra recorded at Tip A and Tip B results in an opposite variation of $\alpha$ and $\beta$ with the resistance ratio. The crossing between the $\alpha$ and $\beta$ curves does not occur exactly at 0.5 . We believe that this is due to different tip apex end atomic scale structure between Tip A and Tip B. It leads to a small difference in the apparent tunnel barrier height estimated from the slope of the corresponding $I_{A}\left(z_{A}\right)$ and $I_{B}\left(z_{B}\right)$ curves already measured when the holder is grounded. Since the metal to metal contact between the back surface of the lead sample and the top surface of stainless steel sample holder is ohmic in our experiment, the lead sample and its holder are at the same $\mu_{S}$ equilibrium chemical potential.

As indicated above, we have also measured $R_{A}=(\pi \hbar /$ $\left.e^{2}\right) \times T_{A S}{ }^{-1}$ and $R_{B}=\left(\pi \hbar / e^{2}\right) \times T_{S B}{ }^{-1}$ independently as a function of Tip A and Tip B apex distance to the $\mathrm{Pb}(111)$ surface by recording their respective $I_{A}\left(z_{A}\right)$ and $I_{B}\left(z_{B}\right)$ characteristics when the holder was grounded. Knowing $R_{A}$ and $R_{B}$ and following (3), we can determine the $V_{S}$ variations based on those data sets as presented in Figure 5b. Notice that when Tip B is exactly at ballistic contact $\left(T_{S B}=1\right.$ or $\left.R_{B}=12.9 \mathrm{k} \Omega\right)$ and $T_{A S}<<1$, then $V_{S}=V_{B}$. This is consistent with the zero shift of the $\mathrm{d} I /$ $\mathrm{d} V$ spectra presented in Figure 2a for a non-zero $V_{B}$. 

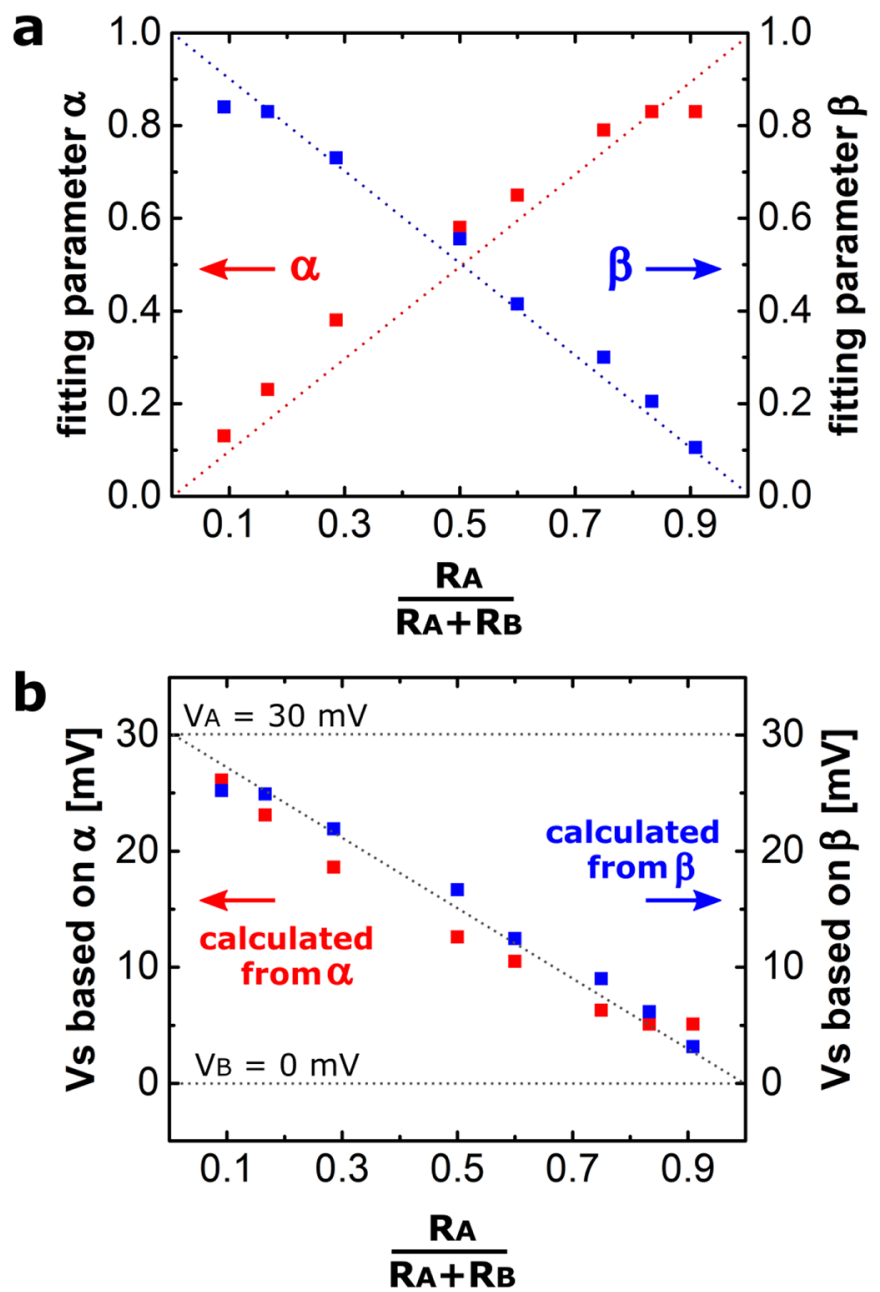

Fig. 5. (a) Variation of the experimental $\alpha$ and $\beta$ obtained in (3) as a function of the $\frac{R_{A}}{R_{A}+R_{B}}$ ratio measured independently with the sample holder grounded. From Figure $3 \mathrm{~d} I / \mathrm{d} V$ spectra series, $a$ and $b$ were obtained going from Figure 3(a)ii and (b)ii series to Figure 3(a)iii and (b)iii series respectively. (b) For a set of $\mathrm{d} I / \mathrm{d} V$ measurements with $V_{A}=30 \mathrm{mV}$ and $V_{B}=0.0 \mathrm{mV}, \quad V_{S}$ is obtained from (3) using the corresponding experimental $\alpha$ and $\beta$ values as a function of the $\frac{R_{A}}{R_{A}+R_{B}}$ ratio. (Each $\frac{R_{A}}{R_{A}+R_{B}}$ data point is corresponding to a $10 \mathrm{pm}$ relative tip height change, Tip A in red and Tip B in blue).

\section{Conclusion}

The floating chemical potential of a lead superconducting metallic sample have been determined at LHe temperature, by transferring electrons through the $\mathrm{Pb}(111)$ surface of this sample between 2 STM tips (the source and the drain electrodes) in a three electrodes configuration where the end atom apex atomic orbitals of each tip are not hybridized with the ones of the uppermost $\mathrm{Pb}(111)$ surface atoms. The source and drain vacuum tunneling junction resistances with the $\mathrm{Pb}(111)$ surface were controlled by tuning the tip apex end atom distance to the $\mathrm{Pb}(111)$ surface with a few picometers precision. The decoherence and relaxation effects coming from those vacuum tunneling junctions and the non-superconducting metallic support of the lead sample has been demonstrated. This support is playing the role of a third electrode. Not grounded, it can be considered as an electron reservoir, the chemical potential of which can be balanced quantum mechanically between the source and drain chemical potentials by a fine tuning of the source and drain atomic scale tunneling contact resistance to the $\mathrm{Pb}(111)$ surface.

\section{Authors contribution statement}

We-Hyo Soe carried out the majority of the experimental work and Christian Joachim of the measurement interpretation. All authors contributed to discussions and writing manuscript. The authors thanks to Takashi Uchihashi from MANA-NIMS for constructive discussion about surface superconductivity, to Erik Dujardin and David Martrou from PicoLab at GNS-CEMES for their support during this work.

\section{References}

1. M. Büttiker, Phys. Rev. B 33, 3020 (1986)

2. M. Büttiker, Phys. Rev. B 35, 4123 (1987)

3. R. Voss, R.B. Laibowitz, A.N. Broers, Appl. Phys. Lett. 37, 656 (1980)

4. S. Washburn, IBM J. Res. Dev. 32, 335 (1988)

5. R. de Piccioto, H.L. Stormer, L.N. Pfeiffer, K.W. Baldwin, K.W. West, Nature 411, 51 (2001)

6. M. Fujimori, S. Hieke, Y. Tarada, T. Hashizume, Nanotechnology 15, S333 (2004)

7. T.N. Tun, M.H.T. Lwin, H.H. Kim, N. Chandrasekar, C. Joachim, Nanotechnology 18, 335301 (2007)

8. T. Ushihashi, U. Ramsperger, T. Nakayama, M. Aono, Jpn. J. Appl. Phys. 47, 1797 (2008)

9. S. Egger, A. Ilie, Y. Fu, J. Chongsathien, D.J. Kang, M.E. Welland, Nano Lett. 5, 15 (2005)

10. C. Joachim, D. Martrou, M. Rezeq, C. Troadec, D. Jie, N. Chandrasekhar, S. Gauthier, J. Phys: Condens. Matter. 22, 084025 (2010)

11. R. Thamankar, T.L. Yap, K.E.J. Goh, C. Troadec, C. Joachim, Appl. Phys. Lett. 103, 083106 (2013)

12. J. Baringhaus, M. Ruan, F. Edler, A. Tejeda, M. Sicot, A.T. Ibrahimi, A. Ping Li, Z. Jiang, E.H. Conrad, C. Berger, C. Tagenkamp, W.A. de Heer, Nature 506, 349 (2014)

13. B. Voigtlander, V. Cherepanov, S. Korte, A. Leis, D. Cuma, S. Just, F. Lüpke, Rev. Sci. Inst. 89, 101101 (2018)

14. O. Kubo, Y. Shingaya, M. Nakaya, M. Aono, T. Nakayama, Appl. Phys. Lett. 88, 254101 (2006)

15. M. Kolmer, P. Brandimarte, J. Lis, R. Zuzak, S. Godlewski, H. Kawai, A. Garcia-Lekue, N. Lorente, T. Frederiksen, C. Joachim, D. Sanchez-Portal, M. Szymonski, Nat. Commun. 10, 1573 (2019)

16. L. Lafferentz, F. Ample, H. Yu, S. Hetch, C. Joachim, L. Grill, Science 323, 1193 (2009)

17. C. Joachim, J.K. Gimzewski, R.R. Schlittler, C. Chavy, Phys. Rev. Lett. 74, 2102 (1995)

18. J. Yang, D. Sordes, M. Kolmer, D. Martrou, C. Joachim, Eur. Phys. J. Appl. Phys. 73, 10702 (2016)

19. J.M. Byers, M.E. Flatté, Phys. Rev. Lett. 74, 306 (1995)

20. M. Schmid, W. Hebenstreit, P. Varga, S. Crampin, Phys. Rev. Lett. 76, 2298 (1996) 
21. S.H. Pan, E.W. Hudson, J.C. Davis, Appl. Phys. Lett. 73, Open Access This article is distributed under the terms of the $2992(1998)$

22. G. Binnig, H. Rohrer, Ch. Gerber, E. Weibel, Phys. Rev. Lett. 49, 57 (1982)

23. M. Büttiker, IBM J. Res. Develop. 32, 317 (1988) Creative Commons Attribution License https://creativecom mons.org/licenses/by/4.0 which permits unrestricted use, distribution, and reproduction in any medium, provided the original author(s) and source are credited.

Cite this article as: We-Hyo Soe, Corentin Durand, Christian Joachim, Low temperature two STM tip tunneling measurements of a floating chemical potential $\mathrm{Pb}(111)$ surface, Eur. Phys. J. Appl. Phys. 87, 31001 (2019) 